\title{
Insect hearing: from physics to ecology
}

\author{
Bernhard Ronacher $\cdot$ Heiner Römer
}

Received: 1 November 2014 / Revised: 10 November 2014 / Accepted: 11 November 2014 / Published online: 21 November 2014 (C) Springer-Verlag Berlin Heidelberg 2014

\begin{abstract}
Almost exactly 100 years ago, Johann Regen performed an ingenious simple experiment where he arranged a male cricket calling in one room and transmitted its song via telephone to another room. There a female could be attracted to the earpiece of a second telephone (Regen 1913). Given the poor frequency characteristics of the early (and contemporary) telephones, one could already speculate about the nature of (temporal) cues that guided the female's phonotactic approach. In the meantime a tremendous progress has been made regarding our understanding of insect hearing (Gerhardt and Huber 2002; Hedwig 2013). This is partly due to the bewildering variety of insect ears having evolved independently many times, and virtually anywhere on the insect body such as on the tibia, abdomen, thorax, wing, mouthparts or the base of the neck (reviews: Hoy and Robert 1996; Yack 2004; Strauß and Stumpner 2015). Insect hearing organs exist as two basic forms: (1) either as tympanal ears with a thin cuticular membrane, an air-filled cavity behind it and a chordotonal organ directly or indirectly coupled mechanically to the tympanum (Robert and Hoy 1998) — these ears respond to sound pressure changes; or (2) as nontympanal hearing organs which respond to the air particle velocity. These are represented by filiform hairs or antennae, such as those in mosquitoes or fruit flies. The collection of articles in this special issue of JCP-A covers a
\end{abstract}

B. Ronacher

Department of Biology, Behavioral Physiology Group, Humboldt-Universität zu Berlin, 10099 Berlin, Germany e-mail: bernhard.ronacher@rz.hu-berlin.de

H. Römer ( $\square)$

Institute for Zoology, Karl-Franzens-University, 8010 Graz, Austria

e-mail: heinrich.roemer@uni-graz.at broad range of anatomical variation in both types of hearing organs.

As Regen's experiment with field crickets already indicates, hearing research has strongly benefited from the fact that many species of insects respond so reliably to acoustic playbacks, and that they do so under very different experimental paradigms, from open-loop laboratory conditions on a trackball to the disturbed and noisy conditions in the field. Moreover, with the advent of neurophysiological techniques hearing and sound communication in insects has become one of the classical areas in neuroethology, aiming to understand the proximate mechanisms of behavior in signalers and receivers (Huber et al. 1989; Gerhardt and Huber 2002; Greenfield 2002; Hedwig 2013). Finally, the refinement of methods such as scanning Laser Doppler Vibrometry for measuring sound-induced vibrations down to the nanometer range has greatly improved our understanding of the biophysics of hearing in recent years.

This issue of JCP-A presents a synopsis of what is currently known about insect hearing. Its title, Insect hearing: from physics to ecology, reflects its broad thematic scope. We have to keep in mind that natural selection may act on virtually all aspects of hearing, from molecules associated with the transduction and amplification process in receptor cells, to sound guides that provide directionality in the small insect receivers, or to the complex behavior in large choruses of singing insects. The contributions to this issue are guided by four basic themes: (1) ears and receptor mechanisms, (2) pattern recognition and directional hearing, (3) ecology of sound communication, and (4) evolution.

Since the first recordings from locust auditory receptor neurons it was established that locusts' ears are, in principle, able to discriminate different frequencies. However, the biophysical and or physiological basis of this capacity 
remained a matter of debate for several decades (Michelsen 1971). Thanks to refined methods of scanning Laser Doppler Vibrometry, it has recently been shown that-similar to vertebrate ears - traveling waves are the basis of frequency discrimination not only in locusts but also in crickets and bush crickets (see Montealegre-Z and Robert 2015). There are remarkable additional similarities between insect and vertebrate hearing: the impedance matching between air and liquid and active amplification processes that enhance tympanal movements or vibrations of the antennae. The latter are essential for the exquisite sensitivity of insect ears that matches that of the most sensitive vertebrate ears (Mhatre this volume, see also Robert and Göpfert 2002). Such rather unexpected functional similarities in differently organized auditory systems point at the similarity of very strong selective pressures (Montealegre and Robert 2015; Mathre 2015). Remarkably, whereas in cochlear outer hair cells electromotility and amplification are mediated by Prestin, in Drosophila the Prestin homologue is not essential for auditory amplification (Kalvie et al. 2015). Instead in flies the mechanotransducer channel NompC (=TRPN1) is required for auditory amplification. Kavli et al. conclude that the advent of Prestin (and the concurring loss of NompC) in vertebrates marks an important evolutionary transition from a transducer-based to a Prestin-based mechanism of auditory amplification. Insect and vertebrate ears thus are examples where different biophysical and molecular solutions have been found to achieve the same goals.

The exquisite sensitivity of ears is combined with a huge range of sound intensities that must - and can-be perceived. The enormous intensity range poses an equally large problem for sensory processing with its limited range of neuronal responses. Range fractionation can relieve this problem to a certain extent, but the ubiquitous solution is neuronal adaptation. It starts with gain control in the sensory neurons, that is by controlling the above-mentioned amplification processes (see Mathre 2015), and is further elaborated in interneurons (Hildebrandt et al. 2015). Hildebrandt et al. take advantage of the multitude of independently evolved auditory pathways found in insects which allowed them to abstract from specific physiological mechanisms and to derive a few general computational principles important for efficient auditory processing. Besides the control of sensitivity by adaptation, they identify three additional computational principles that should be realized within any peripheral auditory pathway.

Two major functions of ears are the detection of friends and foes; a third, less prominent aspect may be the detection of prey (see parasitoids, below). All of these aspects require not only the evaluation and interpretation of sound signals ("recognition" of cues or signals) but also the ability to localize the sound source. Directional information is delivered "for free" in particle velocity receivers like the antennae of mosquitoes or Drosophila since the velocity vector is inherently directional. However, for small animals relying on tympanic ears as, e.g., grasshoppers, crickets, katydids, cicadas, and mantises, sound localization becomes a problem: the small ear distances create only minute interaural time differences, and the ratio of body size to sound wavelength makes substantial interaural intensity differences due to diffraction unlikely. The basic solutions for this problem are pressure gradient receivers and the use of high frequency (small wavelength) communication sounds. The article by Römer (2015) focuses on the evolution of pressure gradient receivers and their function in outdoor hearing. Other rarely considered aspects are the contribution of directional hearing to auditory scene analysis, and directionality in the third dimension, being essential for many insects in their complex habitat. Directional information is further enhanced by contralateral inhibition, which is another general motif of peripheral auditory processing (Hildebrandt et al. 2015).

To use and interpret acoustic communication signals in the context of mate finding is one of the major functions of hearing systems-although it may not have been their original task in the past. Despite the ability to discriminate frequencies or to establish frequency filters tuned to the main frequency content of communication signals, the pattern of amplitude modulations has been found to be most critical for signal recognition in many insect taxa. Following different approaches, two articles in the present issue of JCP-A focus on how crickets and grasshoppers process the temporal patterns of signals. Ronacher et al. (2015) present a linear-nonlinear ( $\mathrm{LN})$ model for song recognition that starts from behavioral tests and describes the behavioral preference functions with a high predictive power, both for grasshoppers and crickets. Most interestingly, this model is able to explain a variety of preference functions found in crickets and katydid species with only slight modifications of inhibitory and excitatory inputs (see also Clemens and Hennig 2013; Hennig et al. 2014). Kostarakos and Hedwig (2015) identified brain neurons in crickets with response patterns that strongly parallel the behavioral preference functions. Most remarkably, the brain recordings reveal different processing steps which fit well to the predictions of the independently developed LN-model.

Ultrasound emitting bats are a major threat to nocturnal insects, which favored the evolution of ultrasound-sensitive ears to escape bat predation in many insect taxa. The paper by Pollack (2015) focuses on common themes and neuronal attributes associated with the central neuronal processing of ultrasound stimuli, and the neuronal circuits enabling effective avoidance in various nocturnal insects. In moths, tympanal hearing organs sensitive to ultrasound have evolved independently at least five times, in response to the advent of echo-locating bats. The existence of ears in 
these taxa later enabled their additional use for communication and promoted the evolution of ultrasound communication signals (Nakano et al. 2015). A close range communication with low-intensity ultrasounds during courtship may be more common in moths than anticipated.

The paper by Lakes-Harlan and Lehmann (2015) looks at predation from the other side and focuses on how parasitoids achieve efficient eavesdropping on their hosts. Two taxa of parasitoid Diptera, the Ormiini and the Emblemasomatini, have evolved tympanal ears independently, which enable a precise localization of the source of communication signals emitted by host insects. In response to strong parasitoid pressure some host species have developed different escape strategies as, e.g., shortening the songs.

The detection and discrimination of signals may be strongly constrained by ecological conditions (Brumm 2013). Whoever has been lucky enough to experience a nocturnal tropical rainforest is overwhelmed by the incredible variety and intensity of sounds. How do animals cope with such severe "cocktail party problems"-although most insects probably do not feel like attendees of a party? Questions related to these problems are the theme of two papers in this special issue. Different solutions for how crickets and katydids avoid masking of their communication signals in multi-species assemblages with high background noise levels are discussed by Schmidt and Balakrishnan (2015). Sharply tuned frequency filters combined with spectral partitioning, spatial release from masking, and spatial separation are powerful general strategies to counter signal masking. Interference and masking by signals of conspecifics pose an additional difficult problem, as both the spectral content and temporal patterns are virtually identical, thus precluding the application of frequency filters or amplitude modulation filters. Greenfield (2015) reviews how individuals in choruses manage to synchronize or alternate their signals, and how a chorus structure may in turn exert selective pressures on the singing of individuals.

Evolutionary aspects are a common background theme in all articles of this special issue as selection acts on all levels, from the stimulus receiver structures and the molecular basis of transduction in sensory cells up to the interaction of individuals in populations. Therefore, we have to ask what were the primary selective forces driving the development and maintenance of ears? The review by Strauß and Stumpner (2015) discusses various constraints that may have led to compromise features of hearing organs and downstream neuronal processing. A particularly interesting sort of constraints becomes evident if we focus on sex differences in hearing systems. Mating preferences as a powerful selection agent are in the focus of the paper by Reinhold and Schielzeth (2015). The authors emphasize that it is necessary to investigate preference functions and choosiness as traits of individuals and not as a population property, to obtain an estimate of the actual selective forces driving the evolution of both signal properties and receiver traits.

Quite a few unexplored avenues remain: for example, how did geographic patterns and phylogenetic lineages support or constrain the evolution of insect hearing and communication systems. It is also puzzling to see that so far hearing has been documented in relatively few orders of insects. As Robert (2005) speculated, the morphological adaptability of insects and the ease with which mechanoreceptive precursors can be used for hearing (Lakes-Harlan et al. 1999; van Staaden and Römer 1998) would suggest that thus far unknown hearing organs may be waiting for discovery in some orders of "atympanate" insects. We hope that this special issue stimulates cross-fertilization between different disciplines, and thus will encourage young scientists to join research in this promising field.

\section{References}

Brumm H (ed) (2013) Animal communication and noise, animal signals and communication 2, Springer, Berlin, Heidelberg. doi:10.1007/978-3-642-41494-7_1

Clemens J, Hennig RM (2013) Computational principles underlying the recognition of acoustic signals in insects. J Comput Neurosci $35: 75-85$

Gerhardt HC, Huber F (2002) Acoustic communication in insects and anurans. University of Chicago Press, Chicago

Greenfield MD (2002) Signalers and receivers: mechanisms and evolution of arthropod communication. Oxford University Press, Oxford

Greenfield M (2015) Signal interactions and interference in insect choruses: singing and listening in the social environment. J Comp Physiol A (in this issue)

Hedwig B (ed) (2013) Insect hearing and acoustic communication. Animal signals and communication 1, Springer, Berlin, Heidelberg. doi:10.1007/978-3-642-40462-7

Hennig RM, Heller K-G, Clemens J (2014) Time and timing in the acoustic recognition system of crickets. Front Physiol 5:1-11. doi $: 10.3389 /$ fphys.2014.00286

Hildebrandt J, Benda J, Hennig M (2015) Computational themes of peripheral processing in the auditory pathway of insects. J Comp Physiol A (in this issue)

Hoy RR, Robert D (1996) Tympanal hearing in insects. Annu Rev Entomol 41:433-450

Huber F, Moore T, Loher W (1989) Cricket behavior and neurobiology. Cornell University Press, Ithaca

Kalvie RG, Fritz JL, Nies F, Göpfert MC, Oliver D, Albert JT, Eberl DF (2015) Prestin is an anion transporter dispensable for mechanical feedback amplification in Drosophila hearing. J Comp Physiol A (in this issue)

Kostarakos K, Hedwig B (2015) Pattern recognition in field crickets: concepts and neural evidence. J Comp Physiol A (in this issue)

Lakes-Harlan R, Lehmann GUC (2015) Parasitoid flies exploiting acoustic communication of insects-comparative aspects of independent functional adaptations. J Comp Physiol A (in this issue)

Lakes-Harlan R, Stölting H, Stumpner A (1999) Convergent evolution of insect hearing organs from a preadaptive structure. Proc R Soc B 266:1161-1167

Mathre N (2015) Active amplification in insect ears: mechanics, models and molecules. J Comp Physiol A (in this issue) 
Michelsen A (1971) The physiology of the locust ear. I. Frequency sensitivity of single cells in the isolated ear. Z Vergl Physiol $71: 49-128$

Montealegre ZF, Robert D (2015) Biomechanics of hearing in katydids. J Comp Physiol A (in this issue)

Nakano R, Takanashi T, Surlykke A (2015) Moth hearing and sound communication. J Comp Physiol A (in this issue)

Pollack J (2015) Neurobiology of acoustically mediated predator detection. J Comp Physiol A (in this issue)

Regen J (1913) Über die Anlockung des Weibchens von Gryllus campestris durch telephonische Übertragung der Stridulation des Männchens. Pflügers Arch Eur J Physiol 155:193-200

Reinhold K, Schielzeth H (2015) Choosiness, a neglected aspect of preference functions: a review of methods, challenges and statistical approaches. J Comp Physiol A (in this issue)

Robert D (2005) Directional hearing in insects. In: Popper AN, Fay RR (eds) Sound source localization. Springer, New York, pp 6-35

Robert D, Göpfert M (2002) Novel schemes for hearing and orientation in insects. Curr Opin Neurobiol 12:715-720
Robert D, Hoy RR (1998) The evolutionary innovation of tympanal hearing in Diptera. In: Hoy RR, Popper AN, Fay RR (eds) Comparative hearing: insects. Springer-Verlag, New York, pp 197-227

Römer H (2015) Directional hearing: from biophysical binaural cues to directional hearing outdoors. J Comp Physiol A (in this issue)

Ronacher B, Hennig M, Clemens J (2015) Computational principles underlying recognition of acoustic signals in grasshoppers and crickets. J Comp Physiol A (in this issue)

Schmidt AKD, Balakrishnan R (2015) Ecology of acoustic signalling in insects. J Comp Physiol A (in this issue)

Strauß J, Stumpner A (2015) Selective forces on origin, adaptation and reduction of tympanal ears in insects. J Comp Physiol A (in this issue)

van Staaden MJ, Römer H (1998) Evolutionary transition from stretch to hearing organs in ancient grasshoppers. Nature 394:773-776

Yack JE (2004) The structure and function of auditory chordotonal organs in insects. Microsc Res Tech 63:315-337 